\title{
Double-Pole Approximation in Time-Dependent Density Functional Theory
}

\author{
H. APPEL, ${ }^{1}$ E. K. U. GROSS, ${ }^{1}$ K. BURKE ${ }^{2}$ \\ ${ }^{1}$ Fachbereich Physik, Freie Universität Berlin, Arnimallee 14, D-14195 Berlin-Dahlem, Germany \\ ${ }^{2}$ Department of Chemistry and Chemical Biology, Rutgers University, 610 Taylor Rd, Piscataway, \\ NJ 08854, USA
}

Received 24 October 2005; accepted 3 January 2006

Published online 15 February 2006 in Wiley InterScience (www.interscience.wiley.com).

DOI 10.1002/qua.20964

\begin{abstract}
A simple approximate solution to the linear response equations of time-dependent density functional theory (TDDFT) is given. This extends the single-pole approximation (SPA) to two strongly coupled poles. The analysis provides both an illustration of how TDDFT works when strong exchange-correlation effects are present and insight into such corrections. For example, interaction can cause a transition to vanish entirely from the optical spectrum. (c) 2006 Wiley Periodicals, Inc. Int J Quantum Chem 106: 2840-2847, 2006
\end{abstract}

Key words: TDDFT; excitation energies; response; oscillator strengths

\section{Introduction}

G round-state density functional theory (DFT) has been very successful for atoms, molecules, and solids $[1,2]$. Similar success is now being enjoyed

Correspondence to: H. Appel; e-mail: appel@physik.fu-berlin.de Contract grant sponsor: EXCITING Research and Training Network of the EU. lence.

Contract grant sponsor: NANOQUANTA Network of Excel-

Contract grant sponsor: Deutsche Forschungsgemeinschaft.

Contract grant number: Sfb658.

Contract grant sponsor: U. S. Department Energy.

Contract grant number: DE-FG02-01ER45928.

Contract grant sponsor: National Science Foundation.

Contract grant number: CHE-03S5405. by time-dependent DFT (TDDFT) [3,4], because of its combination of accuracy combined with low computational cost [5]. While TDDFT has a huge variety of applications [6], it is low-lying photo-excitations of molecules that has seen its greatest use [5].

In the present work, we restrict our discussion to linear response of a nondegenerate ground state. Just as in ground-state DFT, all many-body effects, i.e., exchange and correlation $(\mathrm{XC})$, are contained in a well-defined functional, the XC kernel [7]. In any practical calculation, this functional must be approximated. In most calculations, an adiabatic approximation is made, and the static limit of the kernel is applied. Typical approximations are the adiabatic local density approximation (ALDA) [7] or generalized gradient approximation, or exact exchange [8-10]. The reliability and accuracy of 
these approximations to TDDFT is much less well understood than it is in ground-state DFT.

One can do many calculations on many systems, in order to gain insight into the accuracy and reliability of theory, but it can be much more effective to develop simple approximations to the solution of the TDDFT response problem [11]. A classic example is the single-pole approximation [12], within which TDDFT yields a simple correction to the KS transition frequencies which is just the expectation value of the Hartree-XC kernel on the transition orbitals. While usually accurate [11], the most important feature of this approximation is the insight it yields into the workings of TDDFT. It yields a first approximation to TDDFT effects with almost no extra effort beyond a ground-state calculation, and gives a simple picture for such effects [13]. It has also been shown [11] that, if a transition is only weakly coupled to others in the system, one can use this to estimate the XC kernel itself. Unfortunately, this is rarely the case in practice.

In the present work, we generalize the SPA to a double-pole approximation (DPA), in which we explicitly solve the TDDFT response equations for exactly two transitions. This produces a variety of results beyond that of SPA. Most importantly, one can study TDDFT XC corrections to KS levels when there is strong coupling between levels. But one can also see when SPA fails, and recover Görling-Levy perturbation theory [14] results for the coupling-constant expansion of excited states [11]. DPA has recently been applied successfully to corehole interaction in the x-ray absorption spectroscopy of three-dimensional (3D) transition metals [15].

\section{Double-Pole Approximation}

In the matrix formulation of the TDDFT response equation within the adiabatic approximation, the exact eigenvalues and oscillator strengths can be obtained from the solution of the following eigenvalue problem [16]:

$$
\sum_{q^{\prime}} W_{q q^{\prime}}(\Omega) v_{q^{\prime}}=\Omega^{2} v_{q^{\prime}}
$$

where the matrix $W$ is given by

$$
W_{q q^{\prime}}(\Omega)=\omega_{q}^{2} \delta_{q q^{\prime}}+4 \sqrt{\omega_{q} \omega_{q^{\prime}}} M_{q q^{\prime}}(\Omega)
$$

and

$$
M_{q q^{\prime}}(\Omega)=\int d^{3} r \int d^{3} r^{\prime} \Phi_{q}^{*}(\mathbf{r}) K\left(\mathbf{r r}^{\prime} \Omega\right) \Phi_{q^{\prime}}\left(\mathbf{r}^{\prime}\right) .
$$

Here $\omega_{i}$ is the Kohn-Sham transition frequency, and for single-particle transitions $q(q \equiv k \rightarrow j)$ the shorthand $\Phi_{q}(\mathbf{r}):=\varphi_{k}(\mathbf{r}) \varphi_{j}^{*}(\mathbf{r})$ has been introduced. The kernel $K\left(\mathbf{r}, \mathbf{r}^{\prime}, \omega\right)$ consists of the bare Coulomb interaction and the approximate $X C$ kernel $f_{\mathrm{XC}}\left(\mathbf{r}, \mathbf{r}^{\prime}, \omega\right)$ :

$$
K\left(\mathbf{r}, \mathbf{r}^{\prime}, \omega\right)=\frac{1}{\left|\mathbf{r}-\mathbf{r}^{\prime}\right|}+f_{\mathrm{XC}}\left(\mathbf{r}, \mathbf{r}^{\prime}, \omega\right) .
$$

Atomic units $\left(e^{2}=\hbar=m=1\right)$ are used throughout.

We now solve these equations exactly for a $2 \times 2$ system, i.e., ignoring coupling to all other transitions. To simplify the discussion, we assume a frequency independent kernel and real orbitals, i.e. $M_{q q^{\prime}}=M_{q^{\prime} q}$. Thus, the relation between matrix elements of Casida's equation and the kernel is

$$
W_{i i}=\omega_{i}^{2}+4 \omega_{i} M_{i i}, \quad W_{12}=4 \sqrt{\omega_{1} \omega_{2}} M_{12} .
$$

Next define the average

$$
\bar{W}=\frac{1}{2}\left(W_{11}+W_{22}\right)
$$

and difference

$$
\Delta W=W_{22}-W_{11}
$$

of the diagonal elements. We define a mixing angle by:

$$
\tan \theta=\frac{2 W_{12}}{\Delta W},
$$

choosing the branch between 0 and $\pi$. The eigenvalues can then be written succinctly as

$$
\Omega_{ \pm}^{2}=\bar{W} \pm \frac{1}{2} \frac{\Delta W}{\cos \theta},
$$

while the normalized eigenvectors are

$$
\vec{v}_{+}=\left(\begin{array}{l}
\sin \frac{\theta}{2} \\
\cos \frac{\theta}{2}
\end{array}\right), \quad \vec{v}_{-}=\left(\begin{array}{r}
-\cos \frac{\theta}{2} \\
\sin \frac{\theta}{2}
\end{array}\right) .
$$

The physical oscillator strength can be obtained from the following expression [16]

$$
f_{ \pm}=\frac{2}{3}\left|\vec{x}^{T} S^{-\frac{1}{2}} \vec{v}_{ \pm}\right|^{2},
$$

where

$$
S^{-\frac{1}{2}}=\left(\begin{array}{cc}
\sqrt{\omega_{1}} & 0 \\
0 & \sqrt{\omega_{2}}
\end{array}\right), \quad \vec{x}=\left(\begin{array}{c}
x_{1}^{\mathrm{KS}} \\
x_{2}^{\mathrm{KS}}
\end{array}\right),
$$


and the $x_{j}^{\mathrm{KS}}$ denote dipole matrix elements of KS orbitals. Given that there are only two transitions, we give a geometric meaning to the oscillator strengths. Writing

$$
f_{1}^{\mathrm{KS}}=\sin ^{2} \alpha^{\mathrm{KS}}, \quad f_{2}^{\mathrm{KS}}=\cos ^{2} \alpha^{\mathrm{KS}}
$$

and

$$
f_{-}=\sin ^{2} \alpha, \quad f_{+}=\cos ^{2} \alpha,
$$

we find

$$
\alpha=\alpha^{\mathrm{KS}}-\theta / 2,
$$

i.e., the oscillator strengths are represented by a unit vector in $2 \mathrm{D}$ space, and the coupling merely rotates this vector. Note that the Thomas-ReicheKuhn (TRK) sum rule (sum of the oscillator strengths is 1 ) is obviously preserved.

\section{Single-Pole Approximation}

As mentioned above, the single-pole approximation is a useful approximation to TDDFT results. We recover SPA results by inserting $\theta=0$ in our formulas. Thus

$$
\Omega_{ \pm}^{\mathrm{SPA}}=\sqrt{\bar{W} \pm \frac{\Delta W}{2}}
$$

and the oscillator strengths reduce to their KS values.

We can now study the leading corrections to SPA produced by DPA when the coupling between poles is weak. Writing $\eta=W_{12} / \Delta W$, and assuming $\eta \ll 1$, for the eigenvalues, we find

$$
\Omega \pm=\Omega_{ \pm}^{\mathrm{SPA}} \pm \frac{W_{12}}{2 \Omega_{ \pm}^{\mathrm{SPA}}} \eta+O\left(\eta^{2}\right)
$$

while for the oscillator strengths, we have

$$
\begin{aligned}
& f_{+}=f_{2}^{\mathrm{KS}}+2 \eta \sqrt{f_{1}^{\mathrm{KS}} f_{2}^{\mathrm{KS}}}+O\left(\eta^{2}\right), \\
& f_{-}=f_{1}^{\mathrm{KS}}-2 \eta \sqrt{f_{2}^{\mathrm{KS}} f_{1}^{\mathrm{KS}}}+O\left(\eta^{2}\right) .
\end{aligned}
$$

Note that the corrections to the peak positions are second order in $W_{12}$, while the corrections to $\sqrt{f_{ \pm}}$are first order. Thus SPA is expected to be much better for peak positions than for peak heights.

Last, we point out that this expansion was deduced for the general case in Ref. [11], and used (among other things) to identify coefficients in the
Görling-Levy expansion of excited-state energies. Our results here agree with those, but in the special case of transitions to which DPA applies, yield results that include a resummation of all orders in the adiabatic coupling constant of DFT.

\section{High-Frequency Limit}

So far we have given exact results for the doublepole approximation. However, in many cases where DPA applies, there is a further simplification. Usually the two transitions are closer to each other than any others that couple to the pair. If in addition their frequency difference is small relative to their mean frequency, for both the interacting and KS systems, i.e.,

$$
\bar{\Omega}, \bar{\omega} \gg \Delta \Omega, \Delta \omega,
$$

we find much simpler results, which are very useful for interpretation.

The SPA discussed above reduces to

$$
\Omega_{ \pm}^{\mathrm{SPA}}=\omega_{i}+2 M_{i i}, \quad(i=1,2) .
$$

In fact, the original SPA was applied for just a forward transition, yielding exactly this result [12]. In general, the symmetric result (sometimes called the small-matrix approximation $[17,18])$ is preferable. We use the term SPA to mean the symmetric result throughout this paper.

The mixing angle is given by

$$
\tan \theta=\frac{4 M_{12}}{\Delta \Omega^{\mathrm{SPA}}},
$$

i.e., it is the ratio of the off-diagonal matrix elements of the kernel on the scale of the separation in SPA that matters. We find

$$
\Omega_{ \pm}=\overline{\Omega^{\mathrm{SPA}}} \pm \frac{\Delta \Omega^{\mathrm{SPA}}}{2 \cos \theta} .
$$

SPA yields the correct average position of the two lines, but their splitting is greater than SPA predicts (level repulsion).

\section{Illustrations}

To illustrate our results, consider a weak lowerfrequency transition $\left(\omega_{1}=9 \mathrm{eV}, f_{1}^{\mathrm{KS}}=1 / 10\right)$ and a strong higher-frequency transition $\left(\omega_{2}=12 \mathrm{eV}, f_{2}^{\mathrm{KS}}=\right.$ $9 / 10)$. We imagine these have significant diagonal 


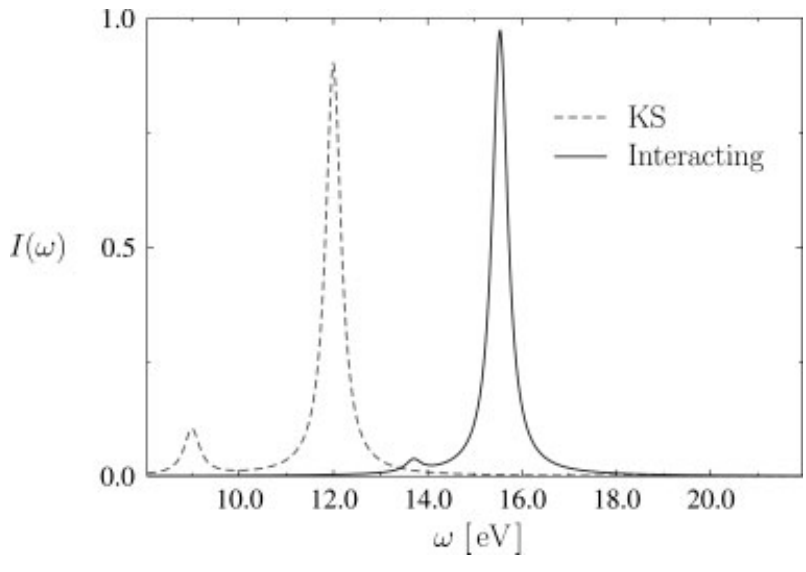

FIGURE 1. Interacting and Kohn-Sham spectra as function of frequency $\left(\omega_{1}=9 \mathrm{eV}, M_{12}=0.2 \mathrm{eV}\right)$.

kernel matrix elements $M_{11}=3 \mathrm{eV}, M_{22}=2 \mathrm{eV}$, but are not strongly coupled to one another, $M_{12}=$ $0.2 \mathrm{eV}$. We have plotted the interacting and $\mathrm{KS}$ spectra in Figure 1. The peaks are Lorentzians of width 0.2 , mimicking a measurement of finite resolution. Because the coupling is weak, the single-pole approximation is excellent and accurately predicts the large shifts in positions. However, SPA wrongly predicts no variation in oscillator strength. In fact, one can see from the figure that the first peak has actually lost intensity relative to its KS value.

In the rest of this section, we explore what happens in the DPA model of TDDFT. To emphasize that it is not the absolute magnitude of the offdiagonal matrix element that is significant, but rather its strength relative to the separation between the peaks, we now consider all the same parameters, but imagine increasing $\omega_{1}$. Figure 2, plots the mixing

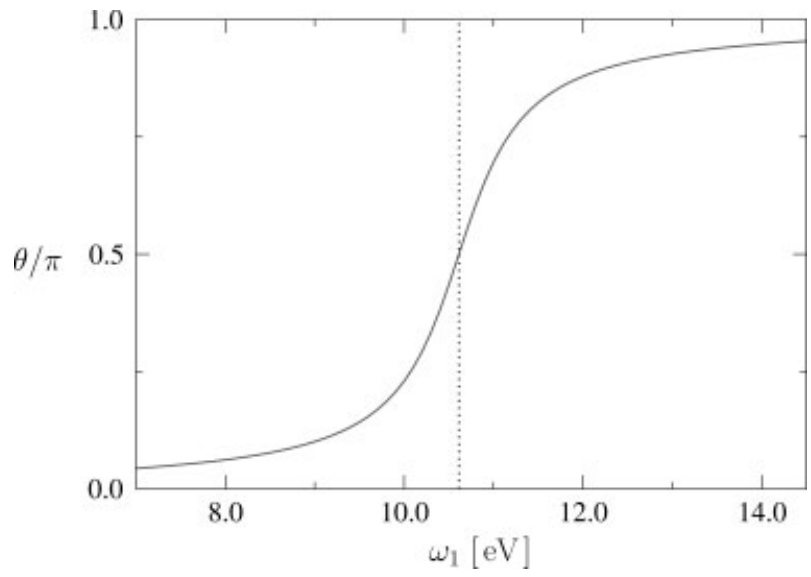

FIGURE 2. Scaled coupling angle $\theta / \pi$ as function of the position of the lower transition. angle as a function of $\omega_{1}$. At $\omega_{c}=2(-3+\sqrt{69}) \mathrm{eV}$ $\approx 10.61 \mathrm{eV}$, the diagonal matrix elements $W_{i i}$ match, so that $\Delta W=0$ and $\theta=\pi / 2$. At that point, the peaks are a 50:50 mixture of the two KS levels. In that region, the levels are strongly coupled, and the spectrum distorts mightily from its KS shape. The width of the transition region can be defined as the change in frequency needed to bring $\theta$ from $\pi / 4$ to $3 \pi / 4$, and, from Eq. (21) in the high-frequency limit, is seen to be $4 M_{12}=0.8 \mathrm{eV}$ here, i.e., proportional to the off-diagonal element, but quite a bit larger. More significantly, there are tails in the transition that decay extremely slowly with pole separation. On the contrary, SPA yields a function that steps from 0 to 1 at $\omega_{c}$.

To see this, in Figure 3, we plot the interacting levels $\Omega_{ \pm}$as a function of $\omega_{1}$, and observe the avoided crossing. Note that straight line plots, extrapolated from the limits where $\omega_{1}$ is either far above or far below $\omega_{c}$, yield extremely accurate results almost everywhere. This is the SPA result. In fact, from Eqs. (9) and (16), we see that the crossover point is exactly given by SPA. Moreover, in the high-frequency limit, Eqs. (20)-(22) yield

$$
|\Delta \Omega|^{2}=\left|\Delta \Omega^{\mathrm{SPA}}\right|^{2}+16\left|M_{12}\right|^{2} .
$$

So if the off-diagonal matrix elements are small relative to the SPA separation, the true separation is not much greater; the closest the two levels come is a separation of $4\left|M_{12}\right|$, i.e., they never cross.

But in Figure 4, we plot the associated oscillator strengths. The effect of coupling is extremely dramatic. Note first that, for $\omega_{1}$ below the strong coupling region, the bigger peak is enhanced above its KS value, and the smaller one reduced. This is pole

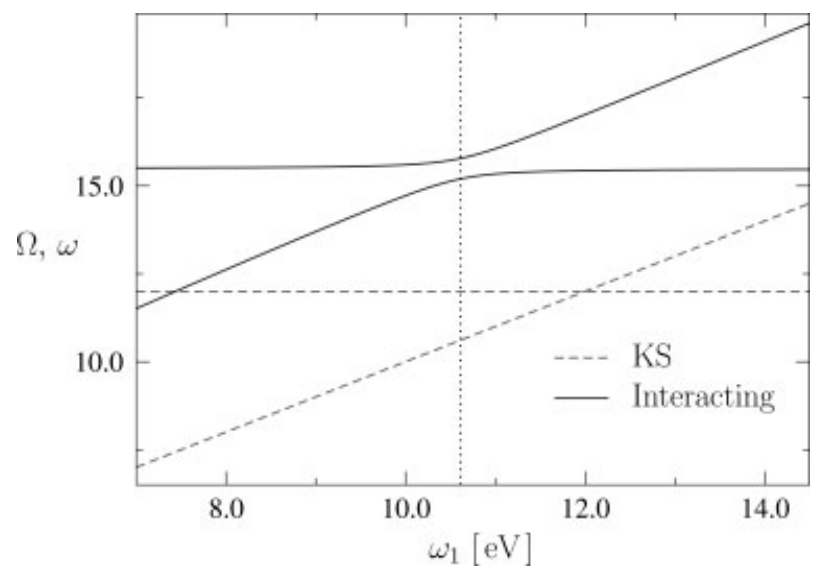

FIGURE 3. Interacting and Kohn-Sham excitation energies as function of $\omega_{1}$. 


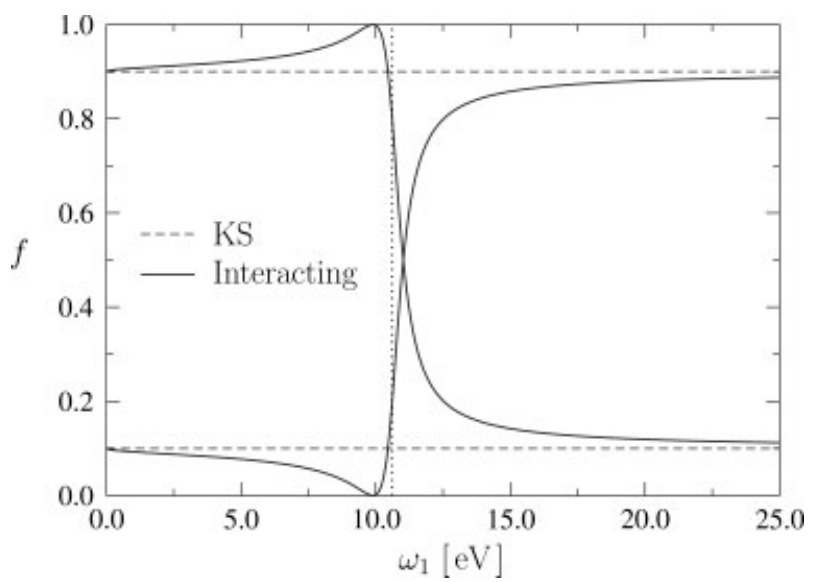

FIGURE 4. Oscillator strengths as function of $\omega_{1}$.

repulsion, and it is even considered very far from the strong coupling region. This effect is entirely missing from SPA. Next we see that there is even a critical value $\omega_{d}$ ( $d$ for dark) at which $f_{-}=0$ exactly. This means the lower peak disappears entirely, and all strength is in the upper peak (Fig. 5). From Eqs. (15) and (21), we find

$$
\Delta \Omega^{\mathrm{SPA}}=g\left(\alpha^{\mathrm{KS}}\right)\left|M_{12}\right| \quad\left(f_{1}=0\right),
$$

where $g(\alpha)=4 / \tan 2 \alpha=16 / 3$ for $f_{1}^{\mathrm{KS}}=0.1$, as is the case here. This yields $8.93 \mathrm{eV}$, whereas the exact result is $9.90 \mathrm{eV}$.

By increasing $\omega_{1}$ just a little more, we come to the position of the avoided crossing $\omega_{c}$ ( $c$ for crossing), where $\theta=\pi / 2$. In fact, Eqs. (13)-(15) yield here

$$
f_{ \pm}=\frac{1}{2} \pm\left\langle f^{\mathrm{KS}}\right\rangle \text {, }
$$

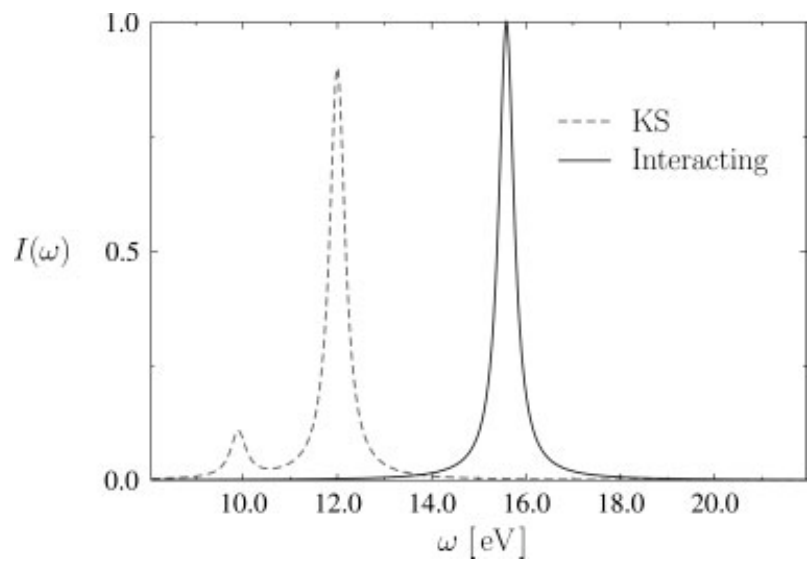

FIGURE 5. Interacting and Kohn-Sham spectra at the critical value $\omega_{1}=\omega_{d} \approx 9.90 \mathrm{eV}$. All intensity is in the upper transition.

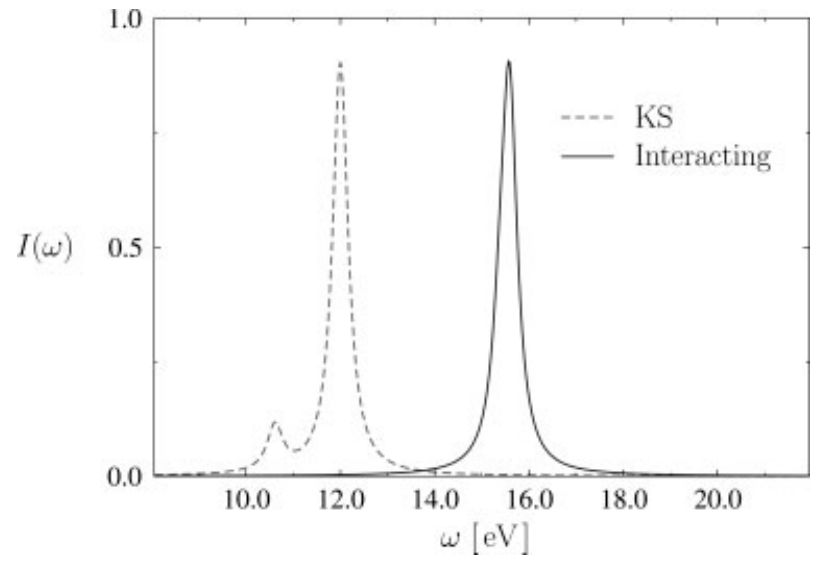

FIGURE 6. Interacting and Kohn-Sham spectra for $\omega_{1}=\omega_{c} \approx 10.61 \mathrm{eV}$.

where $\left\langle f^{\mathrm{KS}}\right\rangle$ denotes the geometric mean, $\sqrt{f_{1}^{\mathrm{KS}} f_{2}^{\mathrm{KS}}}$. In our case, this yields $f_{-}=0.2$ and $f_{+}=0.8$, respectively, giving the lower peak double its $\mathrm{KS}$ weight. Figure 6 shows the spectrum for $\omega_{1}=\omega_{c}$, and observe how much it differs from its KS doppelganger. There appears to be only one peak, but in fact there are still two, although the broadening obscures this. They are very close together.

The final interesting point is $\omega_{e}$ ( $e$ for equal), where the interacting oscillator strengths equal, i.e., both are $1 / 2$. At the equality point, $\alpha=\pi / 4$, and so $\theta=$ $\pi / 2-2 \alpha^{\mathrm{KS}}$. Again using the high-frequency limit, Eq. (21), yields

$$
\Delta \Omega^{\mathrm{SPA}}=-4 M_{12} \cot \left(2 \alpha^{\mathrm{KS}}\right),
$$

i.e., the same distance above the crossing point, as the amount the point $f_{-}=0$ is below. This yields

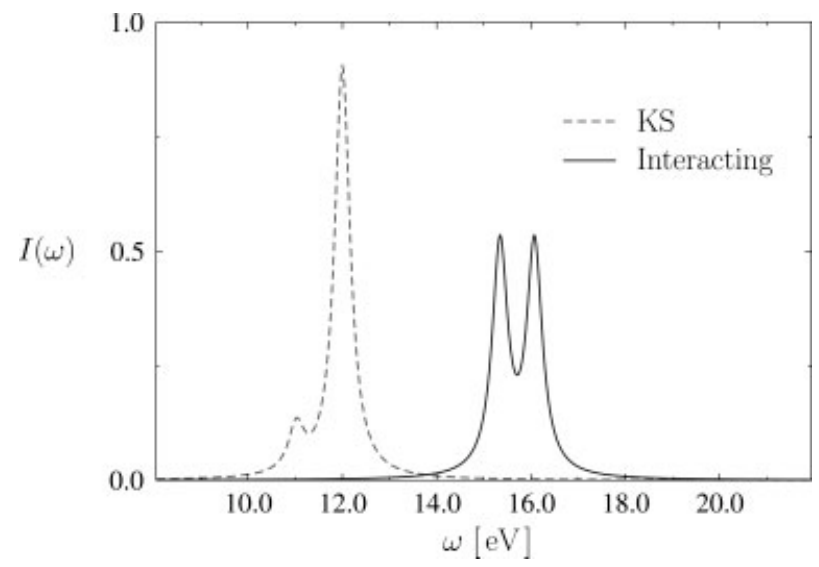

FIGURE 7. Interacting and Kohn-Sham spectra for $\omega_{1}=\omega_{e} \approx 11.02 \mathrm{eV}$, producing equal interacting oscillator strengths. 


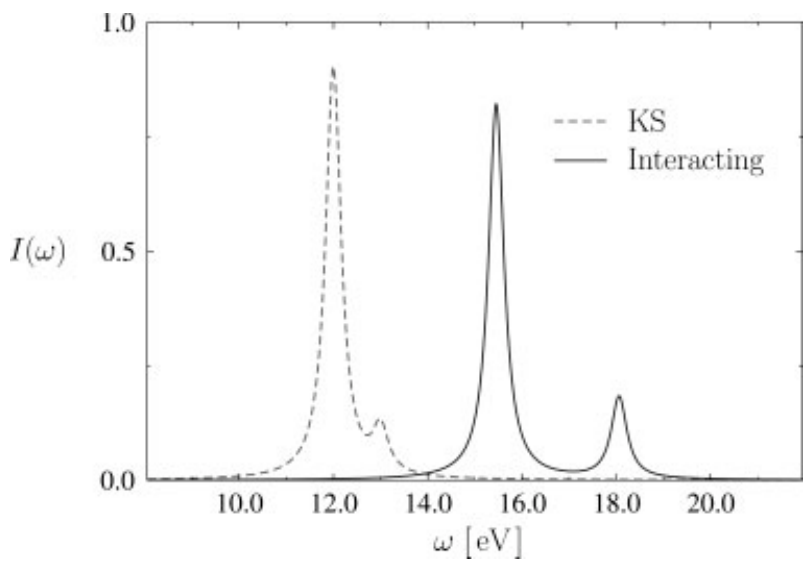

FIGURE 8. Interacting and Kohn-Sham spectra for $\omega_{1}=13 \mathrm{eV}$.

$12.29 \mathrm{eV}$, whereas the exact number is $11.02 \mathrm{eV}$ (Fig. 7).

Finally, in Figure 8, we consider $\omega_{1}=13 \mathrm{eV}$. Now the oscillator strengths have returned (almost) to their KS values, but + and - have been reversed. Lastly, we demonstrate the dependence of these results on the strength of $M_{12}$ relative to the diagonal elements. We have so far presented only the case $M_{12} \ll M_{i i}$. But we have argued that it is only the ratio $\left|M_{12}\right| / \Delta \Omega^{\mathrm{SPA}}$ that matters. Thus, increasing $M_{12}$ does not change the shape of the curves (around the turnover point), but only changes the scale on which the action takes place. In Figure 9, we change $M_{12}$ to $1 \mathrm{eV}$ and $2.5 \mathrm{eV}$, and see this occur. Since the turnover occurs on a scale of about $4\left|M_{12}\right|$, almost the entire region has strong coupling for $M_{12}=2.5 \mathrm{eV}$.

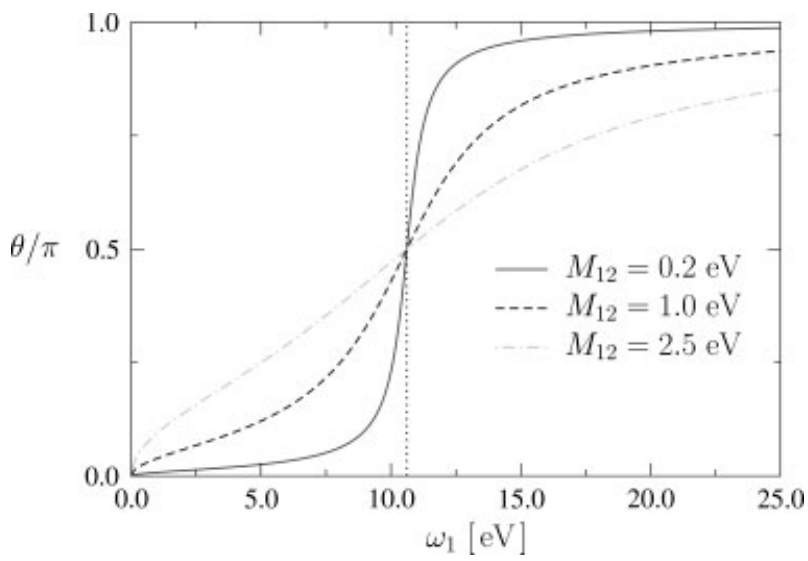

FIGURE 9. Scaled coupling angle $\theta / \pi$ as function of $\omega_{1}$. The plot compares three different regimes for the off-diagonal matrix element $M_{12}$.

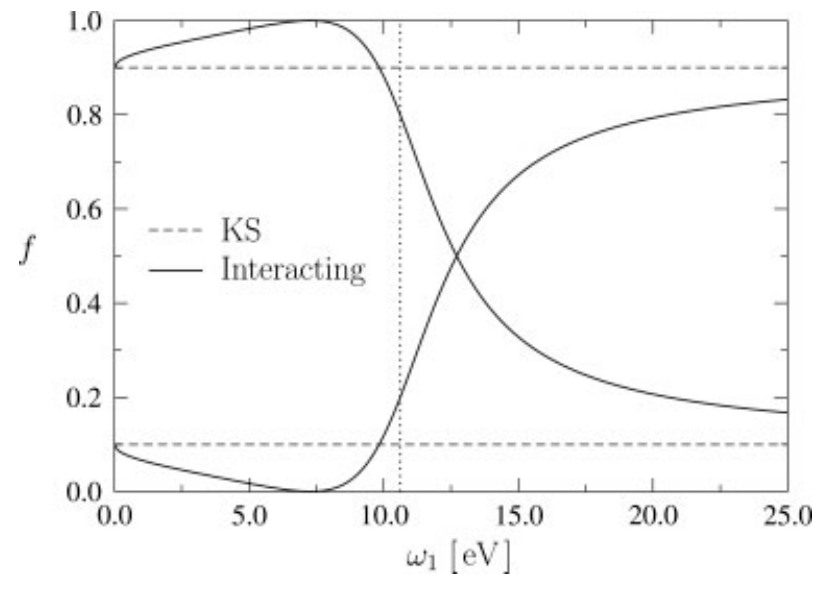

FIGURE 10. Same as Fig. 4, but for the case $M_{12}=1.0 \mathrm{eV}$.

Lastly, we examine this behavior as a function of $M_{12}$. In Figures 10 and 11, we repeat the plot of oscillator strengths versus $\omega_{1}$ for this system, but now with $M_{12}=1 \mathrm{eV}$ and $M_{12}=2.5 \mathrm{eV}$, respectively. We see that the larger values lead to qualitatively similar behavior, but over a broader frequency scale.

\section{Inversion}

The above sections present the TDDFT response equations in the usual manner. First solve the ground-state KS problem, finding occupied and unoccupied levels, then calculate matrix elements of the kernel (with some functional approximation), and calculate the true transitions and oscillator strengths of your system. However, we are

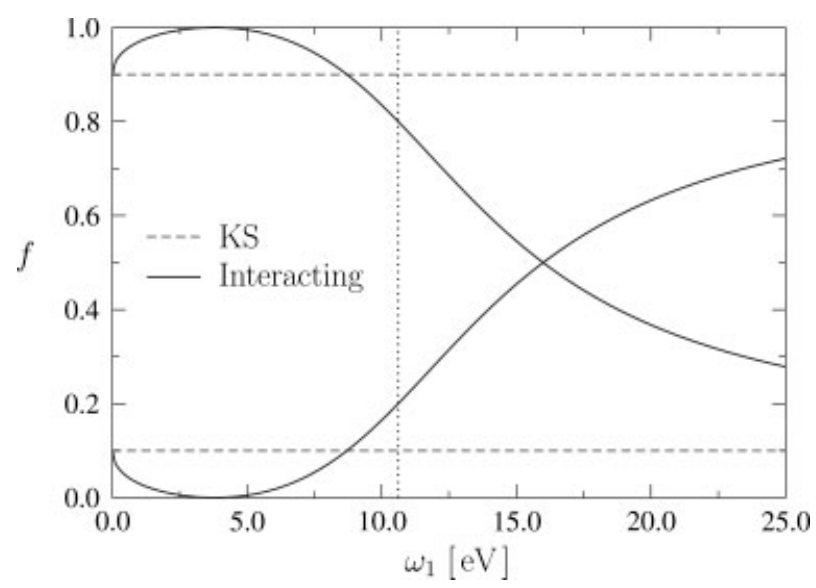

FIGURE 11. Same as Fig. 4, but for the case $M_{12}=2.5 \mathrm{eV}$. 
motivated to gain insight into the excitations, and so we ask the reverse question: Given the experimental spectrum, what can we learn about the kernel? Inverting our equations to solve for $\theta$ yields

$$
\theta=2\left(\alpha-\alpha^{\mathrm{KS}}\right) .
$$

Thus, knowledge of the KS oscillator strengths, combined only with the experimental oscillator strengths, yields the mixing angle, which measures how strongly the transitions are mixed. No knowledge of the positions of transitions is needed.

Solving for the diagonal matrix elements we arrive at

$$
\begin{aligned}
& W_{11}=\overline{\Omega^{2}}-\left(\Delta \Omega^{2} / 2\right) \cos \theta, \\
& W_{22}=\overline{\Omega^{2}}+\left(\Delta \Omega^{2} / 2\right) \cos \theta,
\end{aligned}
$$

where $\overline{\Omega^{2}}$ is the average of $\Omega^{2}$ and $\Delta \Omega^{2}$ is the difference, while the off-diagonal matrix element is

$$
W_{12}=\left(\Delta \Omega^{2} / 2\right) \sin \theta .
$$

Again, the experimental positions combined with the mixing angle are sufficient to determine the elements of the matrix $W$. The kernel matrix elements themselves are then found simply, by using the KS transition frequencies:

$$
M_{j j}=\frac{W_{j j}}{4 \omega_{j}}-\frac{\omega_{j}}{4}
$$

and

$$
M_{12}=\frac{\Delta \Omega^{2} \sin \theta}{8 \sqrt{\omega_{1} \omega_{2}}} .
$$

These equations provide an exact way to recover the matrix elements $W_{i j}$ of the original matrix and therefore the matrix elements $M_{i j}$ of the kernel $K$ solely from the knowledge of the eigenvalues and the angle $\theta$.

While the above formulas are completely general, in practice strong coupling tends to occur between neighboring transitions. In those cases, the differences between the two transition frequencies are often much smaller than the transition frequencies themselves. Thus we expand in the small parameter $\Delta \Omega / \bar{\Omega}$, to find

$$
\begin{aligned}
& W_{11}=\bar{\Omega}(\bar{\Omega}-\Delta \Omega \cos \theta), \\
& W_{22}=\bar{\Omega}(\bar{\Omega}+\Delta \Omega \cos \theta), \\
& W_{12}=\bar{\Omega} \Delta \Omega \sin \theta .
\end{aligned}
$$

To further extract the matrix elements of the kernel, we assume the KS transitions satisfy the same requirement, i.e., that the experimental transitions are close to the KS transitions on the scale of the average transition. This yields

$$
\begin{aligned}
& M_{11}=(\bar{\Omega}-\Delta \Omega \cos \theta) / 4-\omega_{1} / 4, \\
& M_{22}=(\bar{\Omega}+\Delta \Omega \cos \theta) / 4-\omega_{1} / 4, \\
& M_{12}=(\Delta \Omega / 4) \sin \theta .
\end{aligned}
$$

These simple expressions give the matrix elements directly, once the KS and experimental information is known. The mixing angle is determined completely by the oscillator strengths, as in Eq. (27). These expressions were used to analyze $\mathrm{x}$-ray absorption spectra in Ref. [15].

\section{Conclusions}

To summarize, we have presented the exact formulas that arise from a double-pole approximate solution to the TDDFT linear response equations. We have shown how these reduce to the single-pole approximation when the coupling between transitions is weak, and derived the leading terms in this expansion, finding results consistent with those of Ref. [11]. However, with DPA, we can go beyond that work, by considering strong coupling. We also derive simpler expressions that are valid when the transitions are of much higher frequency than the splittings. We illustrated our results, finding that (i) the oscillator strengths can deviate significantly from their KS values, even when the coupling is very weak; (ii) the scale to compare the off-diagonal matrix element to is the splitting in the singlepole approximation, and (iii) the weaker peak even vanishes at a special value of the coupling.

\section{ACKNOWLEDGMENTS}

K. B. acknowledges support of the U. S. Department of Energy under grant DE-FG02-01ER45928, and the National Science Foundation under grant CHE-0355405.

\section{References}

1. Kohn, W.; Sham, L. J. Phys Rev A 1965, 140, 1133.

2. Fiolhais, C.; Nogueira, F.; Marques, M., Eds. A Primer in Density Functional Theory; Springer-Verlag: New York, 2003. 
3. Runge, E.; Gross, E. K. U. Phys Rev Lett 1984, 52, 997.

4. Burke, K.; Werschnik, J.; Gross, E. K. U. J Chem Phys 2005, 123,062206

5. Furche, F.; Rappoport, D. In Computational Photochemistry; Olivucci, M., Ed.; Elsevier: Amsterdam, 2005

6. Marques, M. A. L.; Gross, E. K. U. Annu Rev Phys Chem 2004, $55,427$.

7. Gross, E. K. U.; Kohn, W. Phys Rev Lett 1985, 55, 2850; 1986, $57,923$.

8. Ullrich, C. A.; Gossman, U. J.; Gross, E. K. U. Phys Rev Lett $1995,74,872$

9. Petersilka, M.; Gossmann, U. J.; Gross, E. K. U. In Electronic Density Functional Theory: Recent Progress and New Directions; Dobson, J. F.; Vignale, G.; Das, M. P., Eds.; Plenum: New York, 1998.

10. Görling, A. Phys Rev A 1998, 57, 3433.
11. Appel, H.; Gross, E. K. U.; Burke, K. Phys Rev Lett 2003, 90, 043005.

12. Petersilka, M.; Gossmann, U. J.; Gross, E. K. U. Phys Rev Lett 1996, 76, 1212

13. Wasserman, A.; Maitra, N. T.; Burke, K. J Chem Phys 2005, $122,133103$.

14. Görling, A.; Levy, M. Phys Rev B 1993, 47, 13105.

15. Scherz, A.; Gross, E. K. U.; Appel, H.; Sorg, C.; Baberschke, K.; Wende, H.; Burke, K. Phys Rev Lett 2005, 95, 253006.

16. Casida, M. E. In Recent Developments and Applications in Density Functional Theory; Seminario, J. M., Eds.; Elsevier: Amsterdam, 1996.

17. Vasiliev, I.; Ögüt, S.; Chelikowsky, J. R. Phys Rev Lett 1999, 82, 1919.

18. Grabo, T.; Petersilka, M.; Gross, E. K. U. J Mol Structure (Theochem) 2000, 501, 353 